\title{
RESEARCH
}

Open Access

\section{Human tissue-specific MSCs demonstrate differential mitochondria transfer abilities that may determine their regenerative abilities}

Swati Paliwal ${ }^{1,2}$, Rituparna Chaudhuri ${ }^{1}$, Anurag Agrawal ${ }^{3^{*}}$ and Sujata Mohanty ${ }^{1 *}$

\begin{abstract}
Background: Recent studies have demonstrated mesenchymal stem cells (MSCs) as effective mitochondrial donors with therapeutic success in multiple experimental models of human disease. MSCs obtained from different tissue sources such as bone marrow (BM), adipose (AD), dental pulp (DP), and Wharton's jelly (WJ) are routinely used in clinical trials with no known study of their mitochondrial donor capacity. Here, we show for the first time that MSCS derived from different tissue sources have different mitochondrial donor properties and that this is correlated with their intrinsic respiratory states.

Methods: MitoTracker-labeled MSCs were co-cultured with Cell Trace-labeled U87-MG cells or rat cardiomyocytes. Mitochondrial transfer abilities of MSCs were assessed by using flow cytometry analysis and fluorescence imaging.

Mitochondrial reactive oxygen species (mtROS) levels were analyzed by using MitoSOX red-based staining, and mitochondrial respiration parameters were analyzed by using a Seahorse XF Analyzer.

Results: AD-MSCs and BM-MSCs displayed higher mitochondrial transfer than DP-MSCs and WJ-MSCs. Counterintuitively, DP-MSCs and WJ-MSCs were more effective in suppressing mtROS levels in stressed recipient cells than AD-MSCs or BMMSCs. Interestingly, the oxygen consumption rates and intrinsic mitochondrial respiration parameters like ATP levels, basal and maximal respiration, and mitochondrial DNA copy number in donor MSCs showed a highly significant inverse correlation with their mitochondrial donation.

Conclusions: We find that there are intrinsic differences in the mitochondrial respiration, donation capacity, and therapeutic efficacy among MSCs of different tissue origin. MSCs with high mitochondrial respiration capacities are associated with lower mitochondrial transfer but more effective suppression of mtROS in stressed recipient cells. This is most compatible with a model where recipient cells optimally regulate mitochondrial transfer such that they take more mitochondria from MSCs with lower mitochondrial function. Furthermore, it appears to be advantageous to use MSCS such as DP-MSCs or WJ-MSCs with higher mitochondrial respiratory abilities that achieved better therapeutic effect with lower mitochondrial transfer in our study. This opens up a new direction in stem cell therapeutics.
\end{abstract}

Keywords: Mitochondrial transfer, Tissue-specific MSCs, Mitochondrial bioenergetics

\footnotetext{
* Correspondence: a.agrawal@igib.in; drmohantysujata@gmail.com

${ }^{3}$ Molecular Immunogenetics Laboratory and Centre of Excellence for

Translational Research in Asthma \& Lung Disease, CSIR-Institute of Genomics and Integrative Biology, Mall Road, Delhi 110007, India

'Stem Cell Facility, DBT Centre of Excellence for Stem Cell Research, All India

Institute of Medical Sciences, New Delhi 110029, India

Full list of author information is available at the end of the article
}

(c) The Author(s). 2018 Open Access This article is distributed under the terms of the Creative Commons Attribution 4.0 International License (http://creativecommons.org/licenses/by/4.0/), which permits unrestricted use, distribution, and reproduction in any medium, provided you give appropriate credit to the original author(s) and the source, provide a link to the Creative Commons license, and indicate if changes were made. The Creative Commons Public Domain Dedication waiver (http://creativecommons.org/publicdomain/zero/1.0/) applies to the data made available in this article, unless otherwise stated. 


\section{Background}

Mesenchymal stem cells (MSCs) are now routinely used in a number of clinical trials in regenerative medicine. They are easily available from different tissue sources such as bone marrow (BM), adipose (AD), dental pulp (DP), and Wharton's jelly (WJ) [1]. Results from a number of clinical trials have shown that MSCs hold huge promise for amelioration of several diseases for which no effective cure is available to date. However, the choice of parameters for determining the optimum tissue source and donor for efficient successful therapeutic outcome remains understudied.

MSCs function through several mechanisms such as inter-cellular mitochondrial transfer, paracrine effect, and direct differentiation to regenerate damaged tissue $[2,3]$. MSCs have been shown to exert a beneficial effect through mitochondrial transfer in many diseases such as stroke, asthma, and cardiac diseases $[4,5]$. MSCs transfer their healthy mitochondria to repair and rescue dysfunctional mitochondria in damaged cells. Spees et al. demonstrated for the first time that mitochondrial transfer from healthy MSCs could rescue cells with non-functional mitochondria and restore their aerobic respiration [6]. Recent advances have demonstrated that stressed cells release certain environmental cues that trigger mitochondrial transfer from MSCs in co-culture [7-10]. Damaged mitochondria and mitochondrial products such as damage-associated molecular patterns (DAMPs) are released as stress signals during cellular injury. These factors, along with elevated levels of reactive oxygen species levels, signal the MSCs to enhance their bioenergetics and initiate mitochondria donation to injured recipient cells $[10,11]$. A majority of studies have shown that mitochondria transfer from MSCs to damaged cells occurs through tunneling nanotubes that form the inter-cellular connections between the donor and recipient cell [12-15]. Nonetheless, several other modes of mitochondrial transfer such as formation of cell junctions, microvesicles, cell fusion, and transfer of isolated mitochondria can also mediate mitochondrial transfer to repair and regenerate damaged recipient cells [3, 4, 16-19]. Mitochondrial transfer from BM-MSCs has been shown to protect mice from lipopolysaccharide (LPS)-induced acute lung injury [16]. MSCs have also shown the potential to reprogram adult cardiomyocytes to a progenitor-like state through cell fusion and cell-to-cell connection that facilitate mitochondrial transfer in ischemic cardiomyoblasts [17, 20, 21]. An upsurge of recent studies has shown successful therapeutic effectiveness of mitochondrial transfer in regeneration of various recipient cells such as damaged corneal epithelium cells, renal tubular cells, brain cortical cells, neurons, alveolar cells, and cardiomyocytes [4, 5, 9]. MSCs isolated from BM-MSCs, AD-MSCs, WJ-MSCs, and DP-MSCs have all been used for transplantation into patients in many clinical trials $[4,22]$. Studies have shown that these MSCs from these different tissue origins demonstrate the ability to transfer mitochondria to rescue damaged cells and restore respiratory potential in recipients [17, 23]. However, to date, no study has compared the mitochondrial transfer efficiencies of tissue-specific MSCs to injured cells with dysfunctional mitochondria. In this study, we investigate the differential mitochondria transfer abilities of MSCs derived from BM-MSC, AD-MSC, DP-MSC, and WJ-MSC sources to stressed U87-MG cells or rat cardiomyocytes, treated with antimycin. Here, we have also compared the rescue potential of tissue-specific MSCs indicated by their abilities to reduce mitochondrial reactive oxygen species (mtROS) levels in recipient cells under oxidative stress. Mitochondrial parameters such as membrane potential, basal mtROS, mitochondrial biomass, mitochondrial DNA (mtDNA) copy number, and ATP levels were also compared between various tissue-specific MSCs to understand the contributing factor for the differences in mitochondrial transfer abilities. This study suggests that mitochondrial parameters of tissue-specific MSCs are critical variables in determining the optimum tissue source of MSCs for regenerative therapy purposes.

\section{Methods}

Revival and expansion of cryopreserved human MSCs Cryopreserved human BM-MSCs, AD-MSCs, DP-MSCs, and WJ-MSCs ( $\mathrm{n}=4$ or 6 each) were selected. These samples were obtained after receiving prior informed consent from the donors. MSCs at passage 3 were revived in low-glucose culture medium containing Dulbecco's modified Eagle's medium (LG-DMEM) (Gibco, Gaithersburg, MD, USA) containing $10 \%$ fetal bovine serum (HyClone, Logan, UT, USA) supplemented with 1\% Glutamax and Penstrep (Invitrogen, Waltham, MA, USA), incubated at $37{ }^{\circ} \mathrm{C}$ with $5 \% \mathrm{CO}_{2}$. In vitro culture expansion and characterization of MSCs and viability test were carried out in accordance with previously described lab protocol [24]. Cells at 75-80\% confluency were used for further experiments. After revival, the cell sample was diluted in a 1:1 dilution using $0.4 \%$ Trypan blue solution; $10 \mu \mathrm{L}$ of this dilution was loaded in a hemocytometer, and viability was confirmed immediately under microscope.

\section{Characterization of the cultured cells Surface marker analysis through flow cytometry}

Single-cell suspensions of MSCs from all of the sources were prepared in media after detaching the cells from the flask using TrypLE Express. The cells at a concentration of $0.5-1 \times 10^{6}$ per $\mathrm{mL}$ were stained with labeled antibodies for surface markers CD105, CD29, CD73, CD90, HLAI and HLAII, and hematopoetic marker CD34/45. These were incubated at room temperature 
for $1 \mathrm{~h}$. Corresponding isotypes: IgG1 coupled with PE, PECy5, APC, and FITC were used as controls. Characterization of the cultured cells was performed at the third passage. The cells were acquired on a BD LSR II flow cytometer and analyzed by using FACS DIVA software as per Dominici et al., 2006 [25]. Table 1 shows surface marker characterization of representative tissue-specific MSCs.

\section{Trilineage differentiation}

MSCs were induced for trilineage differentiation (osteogenesis, adipogenesis, and chondrogenesis) and cells showed successful differentiation to these three lineages as indicated by specific staining for every lineage [26].

\section{Co-cultures of MSCs with stressed cells}

Tissue-specific MSCs (BM-MSCs, AD-MSCs, DP-MSCs, and WJ-MSCs) were labeled with $100 \mathrm{nM}$ MitoTracker Green FM (Thermo Fisher Scientific, Waltham, MA, USA) in accordance with the protocol of the manufacturer. U87-MG and rat cardiomyocytes were labeled with Cell Trace Violet ${ }^{\mathrm{Tu}}$ (Thermo Fisher Scientific) at a 5- $\mu \mathrm{M}$ concentration in accordance with the protocol of the manufacturer. Two media washes were given to remove any unbound reagent. Tissue-specific MSCs were trypsinized and seeded onto wells containing antimycin-treated U87-MG or rat cardiomyocytes at a 1:1 ratio containing equal amounts of respective media. The percentage transfer of mitochondria from MSCs to stressed recipients was calculated after $24 \mathrm{~h}$ of co-culture. The cells were assessed for various parameters by confocal imaging or flow cytometry analysis.

Table 1 Surface marker characterization of tissue-specific mesenchymal stem cells (expressed in percentages)

\begin{tabular}{lllllllll}
\hline S. no. & Cell type & CD105 & CD73 & CD90 & CD29 & HLA-I & HLA-II & CD34/45 \\
\hline 1 & BM-MSCS & 86.9 & 80 & 91.1 & 72.2 & 85.7 & 0 & 2.5 \\
2 & BM-MSCS & 81.8 & 81 & 90 & 76 & 83.8 & 0.7 & 0 \\
3 & BM-MSCS & 84.6 & 82.9 & 91.8 & 75.9 & 81 & 1.6 & 0 \\
4 & AD-MSCS & 86.7 & 74.9 & 71.2 & 44.5 & 83.6 & 0 & 0 \\
5 & AD-MSCs & 75.2 & 69.8 & 84.5 & 70.9 & 79.2 & 1.1 & 2.2 \\
6 & AD-MSCs & 76.8 & 73.1 & 81.1 & 68.8 & 75.3 & 0.5 & 0 \\
7 & DP-MSCS & 80.6 & 87.5 & 78.1 & 79.6 & 82.5 & 1.1 & 2.2 \\
8 & DP-MSCS & 86 & 85.2 & 83 & 82.1 & 80.7 & 0 & 0 \\
9 & DP-MSCS & 84.3 & 83.3 & 60.9 & 84.4 & 92.2 & 3.3 & 0 \\
10 & WJ-MSCS & 71.6 & 79.4 & 85.2 & 83.3 & 77.2 & 0 & 0.1 \\
11 & WJ-MSCS & 82 & 96.9 & 98.1 & 92.4 & 73.6 & 0.1 & 0 \\
12 & WJ-MSCS & 87 & 96.8 & 98.2 & 92.1 & 73 & 0 & 0 \\
\hline
\end{tabular}

Abbreviations: AD-MSC adipose-mesenchymal stem cell, BM-MSC bone marrow-mesenchymal stem cell, DP-MSC dental pulp-mesenchymal stem cell, WJ-MSC Wharton's jelly-mesenchymal stem cell

\section{Oxidative stress induction}

Antimycin A (Sigma-Aldrich, St. Louis, MO, USA) at $100 \mathrm{nM}$ was added to culture media of U87-MG or cardiac cells $(50,000$ cells per well in a 12-well culture plate) and was further incubated for 16-18 $\mathrm{h}$ to induce oxidative stress prior to adding MSCs in the co-culture system.

\section{Live cell microscopy}

Live cell imaging was carried out by confocal microscope (Leica TCS SP5; Leica, Wetzlar, Germany). Live cell microscopy was carried out in cells seeded in two- or four-chamber glass slides (Nunc ${ }^{\mathrm{Tm}} \mathrm{Lab}^{\mathrm{T}} \mathrm{Tek}{ }^{\mathrm{m}} \mathrm{II}$ Chamber Slides, Thermo Scientific Fisher, USA) under optimal culture conditions, with $37{ }^{\circ} \mathrm{C}$ temperature and $5 \% \mathrm{CO}_{2}$. Cells were imaged $24 \mathrm{~h}$ after co-culture. All images showing mitochondria transfer were captured with either $100 \times$ objective (DMI6000) or 63× lambda blue objective (SP5).

\section{Mitochondria parameters assessment by flow cytometry}

MitoTracker Green FM (excitation/emission: 419/ $560 \mathrm{~nm}$ ) was used for mitochondrial biomass quantification in MSCs. Tetramethylrhodamine ethyl ester perchlorate (TMRE) (excitation/emission: 550/575 $\mathrm{nm}$ ) was used at a concentration of $100 \mathrm{nM}$ and incubated for $20 \mathrm{~min}$ at $37{ }^{\circ} \mathrm{C}$ for assessment of membrane potential of MSCs. mtROS was measured by using MitoSOX red (Thermo Fisher Scientific) at a concentration of $4 \mu \mathrm{M}$ for $20 \mathrm{~min}$ and incubated at $37{ }^{\circ} \mathrm{C}$ with $5 \% \mathrm{CO}_{2}$. Quantification of mtROS was carried out by using a BD LSR II flow cytometer (Becton Dickinson, Franklin Lakes, NJ, USA) with at least 10,000 events for each sample and analyzed with Becton Dickinson FACS Diva (version 6.1.2). Readings (in duplicates) for mean fluorescence intensity (MFI) in the $\mathrm{PE}$ region were recorded in arbitrary units (AU).

\section{Genomic DNA isolation}

Genomic DNA was isolated from tissue-specific MSCs for mtDNA copy number analysis by using a GeneJET Genomic DNA extraction kit (Thermo Fisher Scientific) in accordance with the instructions of the manufacturer; $5 \times 10^{6}$ MSCs were processed for the same.

\section{Measurement of mitochondrial respiration}

MSCs from all four tissue origins used in this study were seeded on 24-well XF-24 plates (Seahorse Biosciences, Billerica, MA, USA) at a population density of about 60,000 per well in DMEM complete media. Oxygen consumption rate (OCR) was measured in all three groups by using the XFe24 Extracellular Flux Analyzer (Seahorse Biosciences). OCR measurements were acquired in the presence of $10 \mathrm{mM}$ glucose before (basal OCR) and after mitochondrial respiration inhibitors were injected in the system. The following inhibitors-9 $\mu \mathrm{M}$ oligomycin, $0.3 \mu \mathrm{M}$ carbonyl cyanide $p$-trifluoromethoxyphenylhydrazone, 
$11 \mu \mathrm{M}$ antimycin, and $11 \mu \mathrm{M}$ rotenone-were used to determine basal respiration, ATP production, maximal respiration, and spare respiratory capacity. These values were normalized for total protein content per well.

ATP levels were analyzed by using an ATP Assay Kit in accordance with the instructions of the manufacturer (Sigma-Aldrich) in the desired samples $\left(5 \times 10^{6}\right.$ cells were used per sample), and colorimetric detection was carried out by using a spectrophotometer (BioTek, Winooski, VT, USA) at $570 \mathrm{~nm}$.

\section{MtDNA copy number assessment}

Real-time polymerase chain reaction (PCR) was performed by using an Eppendorf Thermocycler machine. Genomic DNA at a concentration of 50 ng was used for PCR reaction using Kappa SYBR Master mix $(5 \mu \mathrm{l})$ and primers at a concentration of $0.3 \mu \mathrm{M}$ in a $10 \mu \mathrm{l}$ total reaction mixture. Primers used for mtDNA are forward primer: $5^{\prime}$-CGAA AGGACAAGAGAAATAAGG-3' and reverse primer: 5'-CTGTAAAGTTTTAAGTTTTATGCG-3' and nuclear housekeeping gene beta-actin primers 5'-TCACCCACA CTGTGCCCATCTAGGA-3' and 5'-CAGCGGAAC CGCTCAT TGCCAATGG-3'. For PCR conditions: denaturation at $95^{\circ} \mathrm{C}$ for $5 \mathrm{~min}$ and 40 cycles of denaturation of $15 \mathrm{~s}$ at $95{ }^{\circ} \mathrm{C}$, annealing $20 \mathrm{~s}$ at $51.2{ }^{\circ} \mathrm{C}(\mathrm{mtDNA}) / 57.3^{\circ} \mathrm{C}$ (nuclear beta-actin gene) and extension $15 \mathrm{~s}$ at $68{ }^{\circ} \mathrm{C}$. The analysis of mtDNA copy number of the mtDNA and nuclear DNA was calculated by using threshold cycle $(\mathrm{Ct})$ number. The reactions for each of the tissue-specific MSCs were carried out in duplicate by obtaining the average value of expression of mitochondrial and nuclear genes. Delta $\mathrm{Ct}$ $(\Delta \mathrm{C})$ was calculated by using the following equation: $\mathrm{Ct}$ (mitochondrial gene) - Ct (nuclear gene). Relative mtDNA copy number was calculated by the $2^{\Delta \mathrm{ct}}$ method.

\section{Statistical analysis}

Data are presented as mean \pm standard error of mean. Statistical analyses were performed by using either one-way or two-way analysis of variance (ANOVA), Tukey's post hoc analysis, or unpaired Student's $t$ test. Statistical significance was set at a $P$ value of less than 0.05 to study differences between donor MSCs. Graphs were plotted by using GraphPad PRISM 7.0 or R 2.13.0 (GraphPad Software, La Jolla, CA, USA) [27].

\section{Results}

\section{Mitochondrial transfer from MSCs to U87-MG and rat cardiac cells}

Revival and characterization of cryopreserved tissue-specific MSCs were performed. All MSCs used for the experiments were characterized for specific markers by using flow cytometry (representative data shown in Table 1). The patient-specific details of each tissue source are provided in supplementary data (Additional file 1: Table S1). Successful mitochondria transfer from tissue-specific MSCs to U87-MG cells and cardiomyocytes was observed when MSCs were co-cultured with the recipient cells. A representative image showing transfer of MitoTracker Green-labeled mitochondria from BM-MSCs to U87-MG cells (Fig. 1a) and cardiomyocytes (Fig. 1b) demonstrates transfer of mitochondria from MSCs to injured recipient cells in co-cultures. A representative flow cytometric data plot shows the percentage of recipient U87-MG cells that uptake mitochondria from BM-MSCs (Fig. 1c). We observed efficient labeling of Mitochondria Tracker Green in MSC cultures and Cell Trace Violet in U87-MG cells, and the transfer of mitochondria was confirmed by the presence of dual-positive cells in co-cultures composed of stained MSCs and U87-MG cells.

\section{Differential mitochondrial transfer from tissue-specific MSCs to recipient cells}

The percentage of cells taking up mitochondria from tissue-specific MSCs was assessed by analyzing the percentage of double-positive cells in co-cultures by using flow cytometry plots (Fig. 1), and bar graphs were plotted to demonstrate differential mitochondria transfer (Fig. 2). Mitochondrial transfer from BM-MSCs and AD-MSCs was found to be significantly higher than that of DP-MSCs in U87-MG co-cultures (Fig. 2a). No significant difference was observed between mitochondrial uptake from BM-MSCs and AD-MSCs (Fig. 2a). Accordingly, BM-MSCs and AD-MSCs also transferred significantly higher percentages of mitochondria compared with DP-MSCs and WJ-MSCs in rat cardiomyocyte co-cultures (Fig. 2b). However, no difference was observed in mitochondrial donation, either between BM-MSCs and AD-MSCs or between DP-MSCs and WJ-MSCs (Fig. 2b).

\section{Tissue-specific MSCs differ in their abilities to reduce mtROS levels in rat cardiomyocytes}

MSCs are known to mitigate oxidative damage by lowering mtROS levels and activating anti-oxidant mechanisms for promoting healing and regeneration of damaged cells [4, 28, 29]. We observed that tissue-specific MSCs differed in their abilities to reduce elevated levels of mtROS in cardiomyocytes (Fig. 3). WJ-MSCs and DP-MSCs reduced mtROS more effectively as compared with both BM-MSCs and AD-MSCs in cardiomyocyte co-cultures (Fig. 3b). However, mtROS reduction capabilities of DP-MSCs and WJ-MSCs were found to be comparable, as were those of BM-MSCs and AD-MSCs (Fig. 3b). We further analyzed the data statistically by using the Pearson correlation test to study the correlation between percentage of cells that take up mitochondria and reduction in mtROS. A strong negative correlation between mitochondrial transfer and mtROS reduction was observed between tissue-specific MSCs in MSC-cardiomyocyte co-cultures (Pearson correlation coefficient, r square: -0.78$)(P<0.002)$ (Fig. 3c). This 

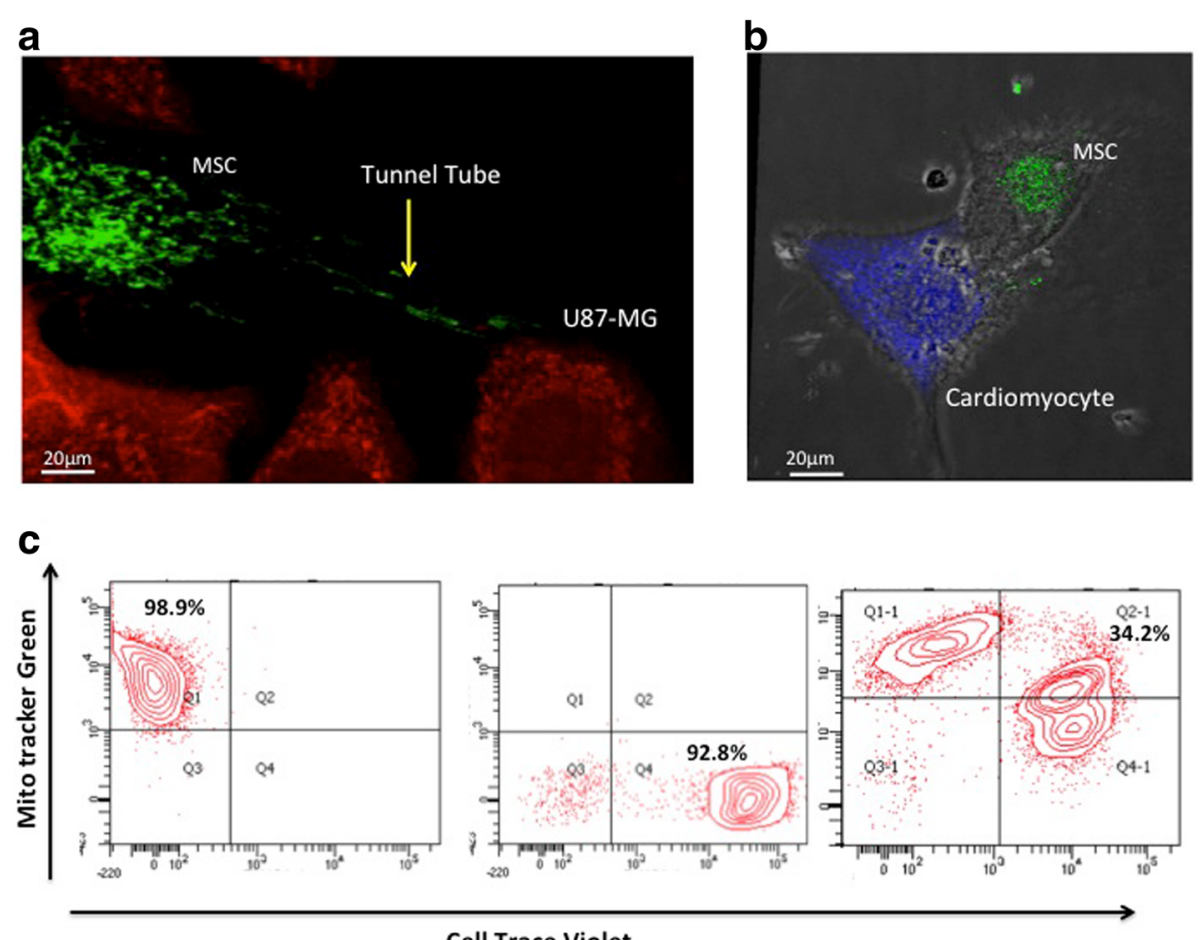

Cell Trace Violet

Fig. 1 Human mesenchymal stem cells (MSCs) can transfer mitochondria to U87-MG cells and rat cardiomyocytes. (a) Representative confocal images of mitochondrial transfer from bone marrow-MSCs (labeled with MitoTracker Green) to U87-MG cells and (b) rat cardiomyocytes (labeled with Cell Trace Violet shown in red and violet, respectively). Scale bar $=20 \mu \mathrm{m}$. (c) A representative flow cytometric data plot shows the percentage of recipient U87-MG cells that take up mitochondria from BM-MSCs. The first plot shows cells stained with only mitotracker labeled MSC cells in Q1 quadrant, second plot shows only cell trace labeled recipient U87-MG cells in Q3 quadrant and third plot shows double positive U87-MG cells in Q2 quadrant

a

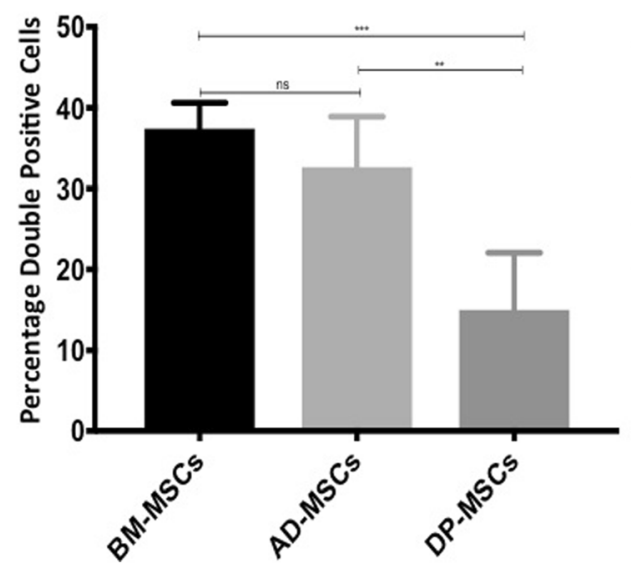

b

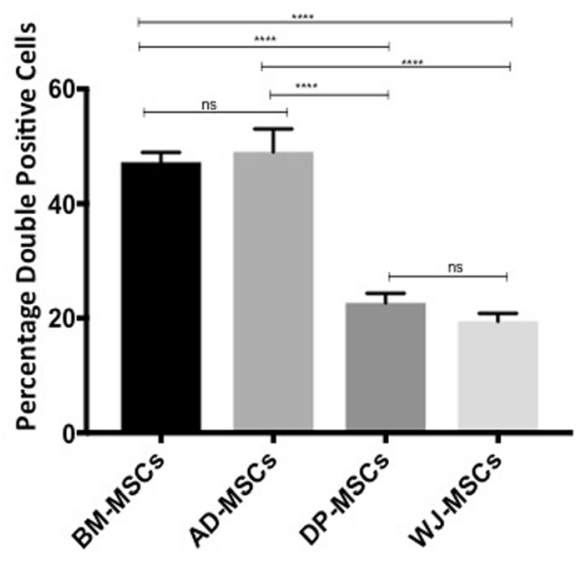

Fig. 2 Tissue-specific mesenchymal stem cells (MSCs) demonstrate differential mitochondrial transfer under oxidative stress. (a) Differential mitochondrial transfer from bone marrow (BM)-MSCS, adipose (AD)-MSCs, and dental pulp (DP)-MSCs is shown in U87-MG determined by flow cytometry. Data are expressed as percentage double-positive cells, mean \pm standard error of the mean (SEM) $(n=4)$, * $P<0.05$, **P $<0.01$, **** $P<0.001$ by one-way analysis of variance (ANOVA) followed by post hoc Tukey analysis. (b) Differential mitochondrial transfer from BM-MSCs, AD-MSCs, DP-MSCs, and Wharton's jelly (WJ)-MSCs is shown in U87-MG determined by flow cytometry. Data are expressed as percentage double-positive cells, mean \pm SEM $(n=4),{ }^{*} P<0.05,{ }^{* *} P<0.01,{ }^{* *} P<0.001$ by one-way ANOVA followed by post hoc Tukey analysis. Abbreviation: $n s$ non-significant 


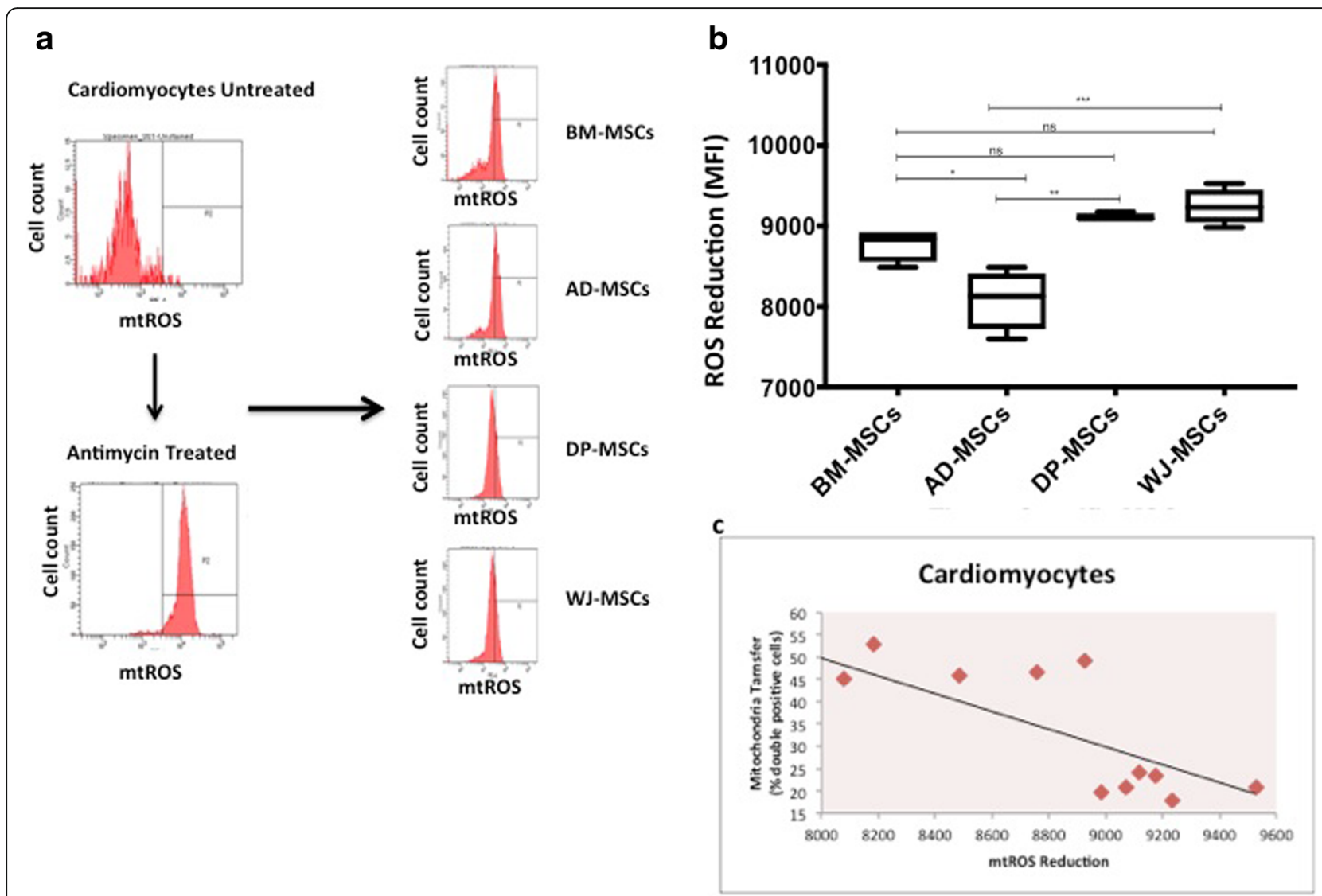

Fig. 3 Differential reduction in reactive oxygen species (ROS) levels by tissue-specific mesenchymal stem cells (MSCs). (a) Representative flow cytometry data depicting mean fluorescence intensity (MFI) of ROS values indicated in arbitrary units in MitoSOX red-stained untreated and antimycin-treated cardiomyocytes, co-cultured with tissue-specific MSCs (left panel). (b) Bar graphs depicting reduction in ROS for bone marrow (BM)-MSCs, adipose (AD)-MSCs, dental pulp (DP)-MSCs, and Wharton's jelly (WJ)-MSCs when co-cultured with stressed cardiomyocytes (right panel) determined by flow cytometry. Data are expressed as mean \pm standard error of the mean (SEM) $(n=4),{ }^{*} P<0.05$, ${ }^{* *} P<0.01,{ }^{* * *} P<0.001$ by one-way analysis of variance (ANOVA) followed by post hoc Tukey analysis. (c) Correlation between reduction in mitochondrial ROS (mtROS) reduction and mitochondrial transfer percentages (percentage double-positive cells) in cardiomyocyte co-cultures determined by Pearson's correlation $(P<0.05)$. Abbreviation: $n s$ non-significant

indicates that although DP-MSCs and WJ-MSCs transferred mitochondria to a smaller number of recipient cardiomyocytes, they brought about higher reduction in overall mtROS levels as compared with BM-MSCs and AD-MSCs in oxidatively stressed cells $(P<0.05)$.

\section{ATP bioenergetics and mitochondrial respiratory parameters} may contribute to differential mitochondrial transfer

We studied mitochondrial copy number to investigate the gene expression of mtDNA with respect to nuclear gene expression to understand the level of mitochondrial biogenesis in various tissue-specific MSCs. We observed that DP-MSCs and WJ-MSCs have significantly higher relative mtDNA copy number compared with BM-MSCs and AD-MSCs (Fig. 4a). To test whether the intrinsic mitochondrial respiration also varies among tissue-specific MSCs, we measured all relevant mitochondrial respiration parameters like ATP production, maximal and spare respiratory capacity, and basal respiration. Interestingly, all parameters were observed to be lower in BM-MSCs and AD-MSCs compared with that in DP-MSCs and WJ-MSCs (Fig. 4b-e). We also analyzed mitochondrial biomass, basal mtROS levels, and mitochondrial membrane potential in tissue-specific MSCs but did not find any significant difference in these parameters (Additional file 2: Figure $\mathrm{S} 1 \mathrm{a}-\mathrm{c}$ ). Corroborating the data shown in Fig. 4, we further found a significant difference in the ATP levels between tissue-specific MSCs using an ELISA (enzyme-linked immunosorbent assay)-based ATP assay (Additional file 2: Figure S1d). DP-MSCs and WJ-MSCs displayed higher ATP levels than BM-MSCs and AD-MSCs (Additional file 2: Figure S1d). Our data confirmed that lower mitochondrial donation took place when the donor MSCs had robust mitochondrial activity in terms of their respiratory capacities (DP-MSCs and WJ-MSCs). This was in contrast to higher mitochondrial 


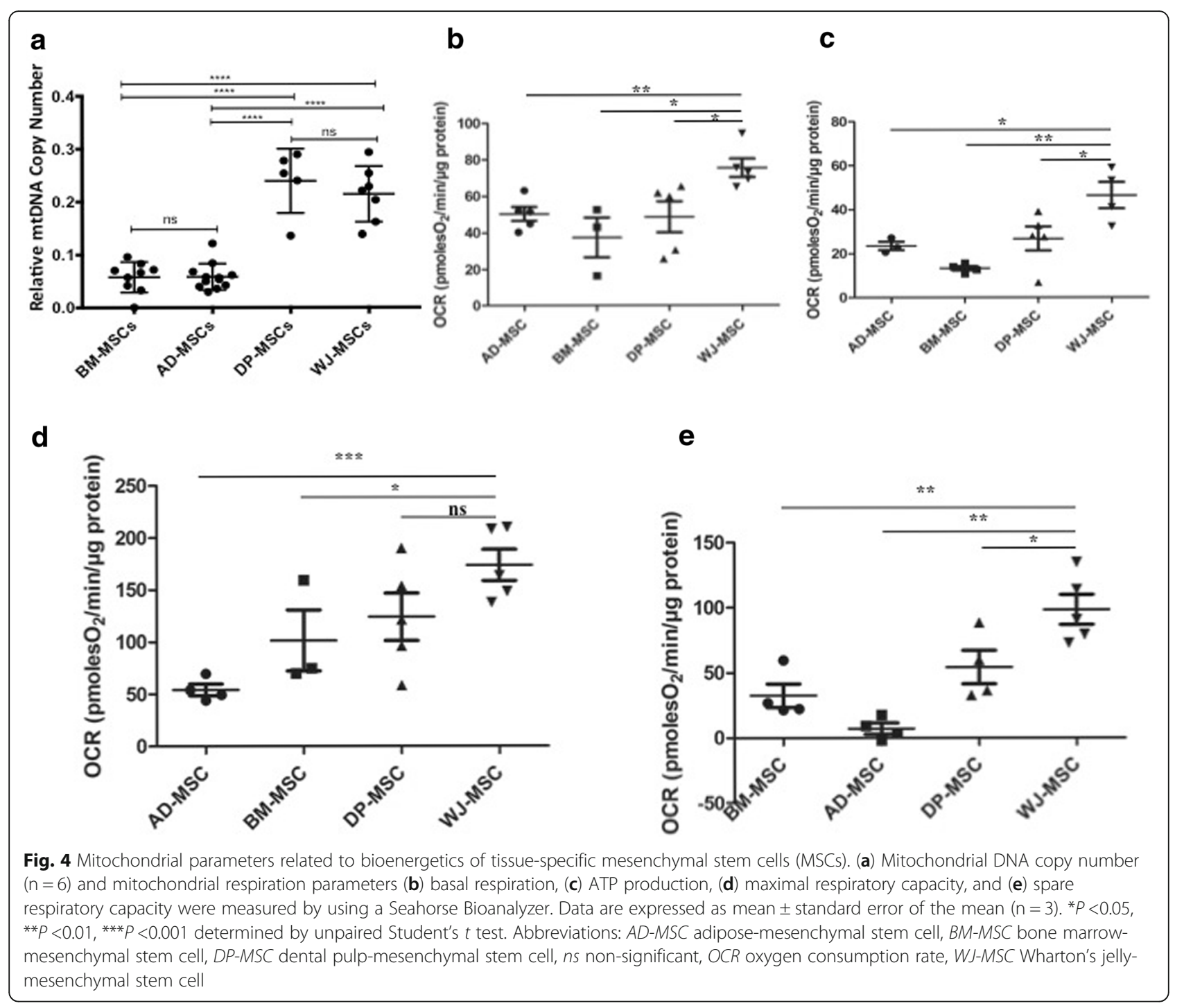

uptake from BM-MSCs and AD-MSCs that demonstrated lower ATP levels and respiratory capacities.

\section{Discussion}

Mitochondrial transfer through MSCs has shown tremendous promise in repair and regeneration of various damaged tissues and in disease models $[4,9,30]$. It has been observed that mitochondrial transfer from MSCs occurs through various modes of transfer such as tunnel tube formation, microvesicles, gap junctions, and cell fusion $[4,5,31]$. The regenerative potential of mitochondria transfer has been observed in cases of asthma, stroke, and myocardial infarction [4, 5, 31]. Although most such studies have used BM-MSCs, other tissue sources like AD-MSCs, DP-MSCs, and WJ-MSCs can also exhibit great regenerative capacities [9, 32]. But regenerative capacity in terms of mitochondrial transfer potential of MSCs obtained from these different tissue sources has not been well explored.

In this study, we compared mitochondria donation capacities of tissue-specific MSCs obtained from different tissue sources and investigated their regenerative potentials. Studies show that MSCs reduce mtROS levels during the repair mechanisms $[4,5,33]$. Thus, we used this parameter as an index of the MSCs' rescue ability. Our study is the first of its kind to explore the relationship between mitochondrial donation capacity and the differential rescue potential of MSCs from different sources.

A potential confounding factor in our study could be that there are age differences among the donor tissue-specific groups; however, these differences reflect the reality of the type of sources and hence could not be eliminated. In addition, we had mixed donors comprising both genders. Since WJ is obtained from the umbilical cord, all donors were female, whereas all of the donors in BM were male. 
Despite having mixed donors (both male and female) in $\mathrm{AD}$ and DP (as shown in Additional file 1: Table S1), we consistently found that mitochondrial donation was lower and intrinsic respiratory capacity was higher in DP and $\mathrm{WJ}$ and vice versa. This indicates that although the gender of the donor can cause large individual differences, our findings with regard to mitochondrial donation capacity and intrinsic respiratory capacity are significant enough to have overridden such individual variations. Unfortunately, it is difficult to obtain MSC donors, so we limited the sample number to between four and six per experiment. However, we believe that our findings will be applicable to a variety of donors with tissue source being the major determining factor.

We have previously shown that differential reduction of reactive oxygen species is observed when human tissue-specific MSCs under oxidative stress are co-cultured with human cardiomyocytes [28]. From our current data, it appears that differences in recipient cell type at the species level do not significantly affect mitochondrial transfer properties of donor MSCs or mtROS reduction potential as similar phenomena are observed from human MSCs to both human cell line and rat cardiomyocyte co-cultures, suggesting that it is a highly conserved and regulated process. Uptake of donor mitochondria by cardiomyocytes under oxidative stress is justified by their need to accept foreign mitochondria to meet the high energy demands of various reparative mechanisms and restore their bioenergetics profile. Relative mtDNA copy number was used to assess the expression levels of genes involved in mitochondrial bioenergetics. Higher mitochondrial respiratory capacities and elevated levels of relative mtDNA level were found in DP-MSCs and WJ-MSCs as compared with BM-MSCs and AD-MSCs. This is in accordance with studies in which an increase in mtDNA copy number suggests the need of gene expression to keep up with high bioenergetics needs in oxidative phosphorylation assembly [34].

Overall, our data suggest that bioenergetics of donor mitochondria may be a critical determining factor regulating differential mitochondrial transfer from tissue-specific donor MSCs. Our data are novel and show that the MSC source significantly determines its mitochondrial donor property and correlates with tissue rescue and intrinsic respiratory states. Thus, mitochondrial parameters and mitochondrial transfer abilities are important parameters that should be considered while choosing an optimum tissue source for regeneration of damaged cells in clinical application.

\section{Conclusions}

Our study showed that tissue-specific MSCs demonstrate differential mitochondria transfer along with differential mtROS reduction in cells under oxidative stress. An inverse relation between mitochondrial transfer and mtROS reduction abilities of tissue-specific MSCs was observed. In addition, higher mitochondrial respiratory capacities were observed in MSCs that donated a lower percentage of mitochondria. It was observed that donor DP-MSCs and WJ-MSCs exhibit higher mitochondrial bioenergetics and robust respiratory capacities with lower mitochondrial transfer compared with BM-MSCs and AD-MSCs. This suggested that MSCs that exhibit higher mitochondrial bioenergetics and robust respiratory capacities are able to achieve higher rescue potential with lower mitochondrial transfer. Thus, this study provides important insight in determining the parameters that should be considered when choosing the optimum source of MSCs for clinical purposes in regenerative medicine.

\section{Additional files}

Additional file 1: Table S1. Age and Gender Details of Tissue-Specific MCSs used in this study. (DOCX $14 \mathrm{~kb}$ )

Additional file 2: Figure S1. Mitochondrial Parameters Assessment (a) mitochondrial biomass determined by Mitotracker green calculated using flow cytometry, $(n=6)$. (b) Mitochondrial membrane potential assessed by Tetramethylrhodamine, ethyl ester (TMRE) using flow cytometry, ( $n=$ 6). (c) ATP assessment confirmation using ATP assay kit (Sigma Aldrich, USA), Data is expressed as mean $\pm \operatorname{SEM}(n=3) .{ }^{*} P<0.05,{ }^{* *} p<0.01,{ }^{* * *} p$ $<0.001$. (JPG $48 \mathrm{~kb})$

\section{Abbreviations}

AD: Adipose; BM: Bone marrow; CD: Cluster of differentiation; Ct: Threshold cycle; DAMP: Damage-associated molecular pattern; DMEM: Dulbecco's modified Eagle's medium; DP: Dental pulp; HLA: Human leukocyte antigen; MSC: Mesenchymal stem cell; mtDNA: Mitochondrial DNA; mtROS: Mitochondrial reactive oxygen species; OCR: Oxygen consumption rate; PCR: Polymerase chain reaction; ROS: Reactive oxygen species; WJ: Wharton's jelly

\section{Acknowledgments}

We thank Sonali Rawat, Suchi Gupta and Rinkey Sharma for characterizing cells used in this study (Stem Cell Facility, AlIMS), Manish Kumar (IGIB, CSIR) for confocal imaging, and Pradeep Rai (BD FACS Academy) and Jamia Hamdard for assistance with flow cytometry.

\section{Funding}

Funds were obtained from the Department of Biotechnology, Ministry of Science and Technology (India).

\section{Availability of data and materials}

Data and materials related to this work are available upon request.

\section{Authors' contributions}

SP performed experiments and analysis for mitochondrial transfer, reactive oxygen species levels, ATP levels, and mtDNA copy number experiments. RC performed Seahorse experiments and their bioenergetics profile analysis. All authors jointly contributed to preparing the manuscript and read and approved the final manuscript. All authors read and approved the final manuscript.

Ethics approval and consent to participate

This study was ethically approved by the Institutional Committee for Stem Cell Research, All India Institute of Medical Sciences (New Delhi). 


\section{Consent for publication}

All authors read the final manuscript and approved the manuscript for publication.

\section{Competing interests}

The authors declare that they have no competing interests.

\section{Publisher's Note}

Springer Nature remains neutral with regard to jurisdictional claims in published maps and institutional affiliations.

\section{Author details}

'Stem Cell Facility, DBT Centre of Excellence for Stem Cell Research, All India Institute of Medical Sciences, New Delhi 110029, India. ${ }^{2}$ Department of Bioscience and Biotechnology, Banasthali Vidyapith, Rajasthan 304022, India. ${ }^{3}$ Molecular Immunogenetics Laboratory and Centre of Excellence for Translational Research in Asthma \& Lung Disease, CSIR-Institute of Genomics and Integrative Biology, Mall Road, Delhi 110007, India.

Received: 27 July 2018 Revised: 7 September 2018 Accepted: 19 September 2018 Published online: 08 November 2018

\section{References}

1. Hass R, Kasper C, Bohm S, Jacobs R. Different populations and sources of human mesenchymal stem cells (MSC): a comparison of adult and neonatal tissue-derived MSC. Cell Commun Signal. 2011:9:12

2. Hayes M, Curley GF, Masterson C, Devaney J, O'Toole D, Laffey JG. Mesenchymal stromal cells are more effective than the MSC secretome in diminishing injury and enhancing recovery following ventilator-induced lung injury. Intensive Care Med Exp. 2015;3(1):29.

3. Caicedo A, Fritz V, Brondello JM, Ayala M, Dennemont I, Abdellaoui N, et al. MitoCeption as a new tool to assess the effects of mesenchymal stem/ stromal cell mitochondria on cancer cell metabolism and function. Sci Rep. 2015;5:9073

4. Ahmad T, Mukherjee S, Pattnaik B, Kumar M, Singh S, Kumar M, et al. Miro regulates intercellular mitochondrial transport \& enhances mesenchymal stem cell rescue efficacy. EMBO J. 2014;33(9):994-1010.

5. Hayakawa K, Esposito E, Wang X, Terasaki Y, Liu Y, Xing C, et al. Transfer of mitochondria from astrocytes to neurons after stroke. Nature. 2016; 535(7613):551-5.

6. Spees JL, Olson SD, Whitney MJ, Prockop DJ. Mitochondrial transfer between cells can rescue aerobic respiration. Proc Natl Acad Sci U S A. 2006;103(5):1283-8.

7. Figeac F, Lesault PF, Le Coz O, Damy T, Souktani R, Trebeau C, et al. Nanotubular crosstalk with distressed cardiomyocytes stimulates the paracrine repair function of mesenchymal stem cells. Stem Cells. 2014;32(1):216-30.

8. Galluzzi L, Kepp O, Kroemer G. Mitochondria: master regulators of danger signalling. Nat Rev Mol Cell Biol. 2012;13(12):780-8.

9. Jiang D, Gao F, Zhang Y, Wong DS, Li Q, Tse HF, et al. Mitochondrial transfer of mesenchymal stem cells effectively protects corneal epithelial cells from mitochondrial damage. Cell Death Dis. 2016;7(11):e2467.

10. Zhang Q, Raoof M, Chen Y, Sumi Y, Sursal T, Junger W, et al. Circulating mitochondrial DAMPs cause inflammatory responses to injury. Nature. 2010; 464(7285):104-7.

11. Mahrouf-Yorgov M, Augeul L, Da Silva CC, Jourdan M, Rigolet M, Manin S, et al. Mesenchymal stem cells sense mitochondria released from damaged cells as danger signals to activate their rescue properties. Cell Death Differ 2017:24(7):1224-38.

12. Abounit S, Zurzolo C. Wiring through tunneling nanotubes--from electrical signals to organelle transfer. J Cell Sci. 2012:125(Pt 5):1089-98.

13. Gerdes HH, Bukoreshtliev NV, Barroso JF. Tunneling nanotubes: a new route for the exchange of components between animal cells. FEBS Lett. 2007; 581(11):2194-201

14. Jackson MV, Morrison TJ, Doherty DF, McAuley DF, Matthay MA, Kissenpfennig A, et al. Mitochondrial transfer via tunneling nanotubes is an important mechanism by which mesenchymal stem cells enhance macrophage phagocytosis in the in vitro and in vivo models of ARDS. Stem Cells. 2016:34(8):2210-23.

15. Rustom A, Saffrich R, Markovic I, Walther P, Gerdes HH. Nanotubular highways for intercellular organelle transport. Science. 2004;303(5660):1007-10.

16. Islam MN, Das SR, Emin MT, Wei M, Sun L, Westphalen K, et al. Mitochondrial transfer from bone-marrow-derived stromal cells to pulmonary alveoli protects against acute lung injury. Nat Med. 2012;18(5):759-65.
17. Acquistapace A, Bru T, Lesault PF, Figeac F, Coudert AE, le Coz O, et al. Human mesenchymal stem cells reprogram adult cardiomyocytes toward a progenitor-like state through partial cell fusion and mitochondria transfer. Stem Cells. 2011;29(5):812-24.

18. Phinney DG, Di Giuseppe M, Njah J, Sala E, Shiva S, St Croix CM, et al. Mesenchymal stem cells use extracellular vesicles to outsource mitophagy and shuttle microRNAs. Nat Commun. 2015;6:8472

19. Pacak CA, Preble JM, Kondo H, Seibel P, Levitsky S, Del Nido PJ, et al. Actindependent mitochondrial internalization in cardiomyocytes: evidence for rescue of mitochondrial function. Biology open. 2015;4(5):622-6.

20. Cselenyak A, Pankotai E, Horvath EM, Kiss L, Lacza Z. Mesenchymal stem cells rescue cardiomyoblasts from cell death in an in vitro ischemia model via direct cell-to-cell connections. BMC Cell Biol. 2010;11:29.

21. Han H, Hu J, Yan Q, Zhu J, Zhu Z, Chen Y, et al. Bone marrow-derived mesenchymal stem cells rescue injured $\mathrm{H} 9 \mathrm{c} 2$ cells via transferring intact mitochondria through tunneling nanotubes in an in vitro simulated ischemia/reperfusion model. Mol Med Rep. 2016;13(2):1517-24.

22. Trounson A, McDonald C. Stem cell therapies in clinical trials: Progress and challenges. Cell Stem Cell. 2015;17(1):11-22.

23. Lin HY, Liou CW, Chen SD, Hsu TY, Chuang JH, Wang PW, et al. Mitochondrial transfer from Wharton's jelly-derived mesenchymal stem cells to mitochondria-defective cells recaptures impaired mitochondrial function. Mitochondrion. 2015;22:31-44

24. Nandy SBMS, Singh M, Behari M, Airan B. Fibroblast growth Factor-2 alone as an efficient inducer for differentiation of human bone marrow mesenchymal stem cells into dopaminergic neurons. J Biomed Sci. 2014:21:83.

25. Dominici M, Le Blanc K, Mueller I, Slaper-Cortenbach I, Marini F, Krause D, et al. Minimal criteria for defining multipotent mesenchymal stromal cells. The International Society for Cellular Therapy position statement. Cytotherapy. 2006;8(4):315-7.

26. Mohanty S, Bose S, Jain KG, Bhargava B, Airan B. TGFbeta1 contributes to cardiomyogenic-like differentiation of human bone marrow mesenchymal stem cells. Int J Cardiol. 2013;163(1):93-9.

27. R Core Team. R: a language and environment for statistical computing. Vienna, Austria: R Foundation for Statistical Computing; 2013. http://www.Rproject.org/

28. Paliwal S, Kakkar A, Sharma R, Airan B, Mohanty S. Differential reduction of reactive oxygen species by human tissue-specific mesenchymal stem cells from different donors under oxidative stress. J Biosci. 2017:42(3):373-82.

29. Hambali Z, Ahmad Z, Arab S, Khazaai H. Oxidative stress and its association with cardiovascular disease in chronic renal failure patients. Indian J Nephrol. 2011;21(1):21-5.

30. Liu K, Ji K, Guo L, Wu W, Lu H, Shan P, et al. Mesenchymal stem cells rescue injured endothelial cells in an in vitro ischemia-reperfusion model via tunneling nanotube like structure-mediated mitochondrial transfer. Microvasc Res. 2014;92:10-8.

31. Masuzawa A, Black KM, Pacak CA, Ericsson M, Barnett RJ, Drumm C, et al. Transplantation of autologously derived mitochondria protects the heart from ischemia-reperfusion injury. Am J Physiol Heart Circ Physiol. 2013; 304(7):H966-82.

32. Yang $X$, Zhang $M$, Zhang $Y$, Li W, Yang B. Mesenchymal stem cells derived from Wharton jelly of the human umbilical cord ameliorate damage to human endometrial stromal cells. Fertil Steril. 2011;96(4):1029-36.

33. Zhang $Y, Y u$ Z, Jiang D, Liang X, Liao S, Zhang Z, et al. iPSC-MSCs with high intrinsic MIRO1 and sensitivity to TNF-alpha yield efficacious mitochondrial transfer to rescue anthracycline-induced cardiomyopathy. Stem Cell Reports. 2016:7(4):749-63.

34. Lee HC, Wei YH. Mitochondrial biogenesis and mitochondrial DNA maintenance of mammalian cells under oxidative stress. Int J Biochem Cell Biol. 2005;37(4):822-34 